\title{
Phosphatidylserine Membrane Translocation in Human Spermatozoa: Topography in Membrane Domains and Relation to Cell Vitality
}

\author{
Malgorzata Kotwicka • Magdalena Jendraszak • \\ Piotr Jedrzejczak
}

Received: 8 January 2011 / Accepted: 11 March 2011 / Published online: 27 March 2011

(c) The Author(s) 2011. This article is published with open access at Springerlink.com

\begin{abstract}
The complex structure of the human spermatozoa membrane comprises five topographic domains. Transmembrane asymmetry of the distribution of phospholipids including phosphatidylserine (PS) is considered a marker of cell activity. The objective of the study was to determine which cytomembrane domains of human spermatozoa are involved in PS membrane translocation and to identify the possible relationship of PS translocation with spermatozoa morphology and vitality. In normozoospermic semen of 35 donors, annexin-V labeling with fluorescein determined PS translocation. Propidium iodide staining distinguished between vital and dead spermatozoa. Three types of PS membrane translocation have been distinguished: (1) in the midpiece, (2) in the acrosomal part and (3) simultaneously in the midpiece and acrosomal part. In morphologically normal vital spermatozoa, PS translocation occurred in the midpiece but never in the equatorial region. In dead spermatozoa, simultaneous PS translocation in the midpiece and acrosomal part was most often observed. The difference between proportions of, respectively, vital and dead spermatozoa presenting PS translocation located in different domains was significant $(P<0.0001)$. In vital cells, there was no difference in PS translocation prevalence between morphologically normal and abnormal spermatozoa $(P>0.05)$. The strict relation of PS translocation to specific membrane domains indicates
\end{abstract}

\footnotetext{
M. Kotwicka $(\varangle) \cdot$ M. Jendraszak

Department of Cell Biology, University of Medical Sciences,

Rokietnicka 5D, 60-806 Poznan, Poland

e-mail: mkotwic@ump.edu.pl

P. Jedrzejczak

Division of Infertility and Reproductive Endocrinology,

University of Medical Sciences, Poznan, Poland
}

functional specificity. It seems doubtful to include this phenomenon in physiological mechanisms of elimination of abnormal spermatozoa.

Keywords Phosphatidylserine membrane translocation . Spermatozoa $\cdot$ Morphology $\cdot$ Apoptosis

\section{Introduction}

Compared to somatic cells, the cell membrane of human spermatozoa is characterized by heterogeneity of its particular fragments (Wolfe et al. 1998). Considering physical, chemical and immunological differences between particular fragments of the human spermatozoa membrane, five domains can be distinguished (Curry and Watson 1995; Ladha et al. 1997). There are three main domains in the head-acrosomal region, equatorial area and post-acrosomal domain - and two domains in the tail-midpiece and main region of the tail.

The mechanisms responsible for retaining a high degree of autonomy of the domains are still poorly understood. There are hardly any evident structural boundaries between domains, apart from the posterior annulus separating the equatorial part from the post-acrosomal part and the annulus ring demarcating the midpiece and the main part of the tail (Ladha et al. 1997). Other regions retain high integrity despite no evident physical boundaries.

It was suggested that high heterogeneity of a spermatozoa cell membrane may result from electrostatic interactions between cytomembrane elements and glycocalyx components which exhibit lateral polarization (Flesch and Gadella 2000). A contribution of lipid rafts to membrane polarization cannot be excluded (Shadan et al. 2004). A crucial role in this phenomenon may be played by 
cytoskeletal elements of a spermatozoa, which also exhibit strong polarization.

Human spermatozoa and somatic cell membranes are similar in respect to their asymmetrical distribution of lipids between the outer and inner cytomembrane layers. Phosphatidylserine (PS) is a membrane phospholipid whose distribution is completely asymmetrical: In normal cells, it constitutes only the inner cytomembrane layer. The mechanisms involved in retaining this PS distribution comprise translocation enzymes and cytoskeletal elements. It has been proven that in particular types of cells (myoblasts, platelets) at some stages of development and activation (prefusion stage of myoblasts, activation of platelets), PS is likely to temporarily translocate from the inner to the outer cytomembrane layer. Moreover, PS translocation (PST) is considered to be one of the early apoptotic markers (Vance and Steenbergen 2005). At present, little is known about the circumstances responsible for PST in spermatozoa. It has not been explained whether this process is a consequence of changes which occur in the spermatozoa cytomembrane during capacitation and the acrosomal reaction or whether it is a result of cellular ageing or the process of pathological spermatozoa elimination.

Our aim was to determine which cytomembrane domains of human spermatozoa are responsible for PS membrane translocation as well as to identify a possible relationship of PST with the vitality and morphology of spermatozoa.

\section{Materials and Methods}

Human semen was collected from 35 healthy donors. Material was taken after sexual abstinence of 2-3 days. Semen analysis was carried out in accordance with the World Health Organization (WHO) guidelines. Samples matched the following criteria: $\geq 20 \times 10^{6}$ spermatozoa/ $\mathrm{ml},>50 \%$ appearing progressively motile, $>15 \%$ demonstrating normal morphology, leukocyte count $<10^{6} / \mathrm{ml}$.

\section{PST}

Annexin-V (AnV)-labeled with fluorescein (AnV-FITC) (Molecular Diagnostics, Darmstadt, Germany) was used to determine PST from the inner to the outer cytomembrane layer. In the presence of calcium ions, AnV selectively bound PS exposed on the cell surface. Simultaneously, propidium iodide (PI) staining (Sigma-Aldrich, St. Louis, MO) was applied to distinguish between viable and dead spermatozoa. Spermatozoa were suspended in an incubation medium containing $10 \mathrm{mM}$ HEPES/NaOH (pH 7.4), $140 \mathrm{mM} \mathrm{NaCl}$ and $5 \mathrm{mM} \mathrm{CaCl} 2$. A cellular suspension was mixed with AnV-FITC and PI (concentrations according to the manufacturer's recommendations); the mixture was incubated at room temperature in the dark for $15 \mathrm{~min}$.

Topohistochemical analysis of PST was performed using a fluorescent microscope (Axioskop 2; Zeiss, Jena, Germany) and a confocal microscope equipped with lens Plan Apochromat $63 \times / 1.4$ Oil DIC (LSM 510, Zeiss). At least 300 spermatozoa were evaluated in each portion of the semen. The evaluation included determination of the PST site as well as vitality and morphology of spermatozoa (according to World Health Organization 1999). Cells were characterized as being either resistant to $\left(\mathrm{PI}^{-}\right)$or susceptible to $\left(\mathrm{PI}^{+}\right)$ PI staining. Also, cells were characterized as being resistant to $\left(\mathrm{AnV}^{-}\right)$or susceptible to $\left(\mathrm{AnV}^{+}\right) \mathrm{AnV}$ binding.

Statistical analysis used the statistical package STATISTICA 7.1 (Statsoft, Tulsa, OK) and GraphPad InSta (GraphPad, San Diego, CA). Nonparametric tests (MannWhitney test, Kruskal-Wallis test with Dunn's post hoc test) were applied to assess differences between proportions of spermatozoa. $P<0.05$ indicated a statistically significant difference.

\section{Results}

Microscopic analysis distinguished four fractions of spermatozoa: (1) $\mathrm{AnV}^{-} / \mathrm{PI}^{-}$, (2) $\mathrm{AnV}^{+} / \mathrm{PI}^{-}$, (3) $\mathrm{AnV}^{-} / \mathrm{PI}^{+}$and (4) $\mathrm{AnV}^{+} / \mathrm{PI}^{+}$and may be interpreted as follows: (1) vital spermatozoa without membrane PST, resistant to PI staining and to AnV binding; (2) vital spermatozoa with membrane PST, susceptible to AnV binding but resistant to PI staining; (3) dead spermatozoa without membrane PST, resistant to AnV binding but susceptible to PI staining; and (4) dead spermatozoa with membrane PST, susceptible to AnV binding and to PI staining (Fig. 1).

The midpiece was the most frequent site of PST. No PST was found in the equatorial region. In some spermatozoa, midpiece PST was accompanied by translocation in the post-acrosomal part. In addition, there was a fraction of spermatozoa with PST in the acrosomal part or simultaneously in the acrosomal and post-acrosomal parts and in the midpiece. Therefore, for further statistical analysis, three types of PST were distinguished: (1) in the membrane of the midpiece region, (2) in the acrosomal part and (3) simultaneously in the acrosomal part and in the midpiece. Since post-acrosomal translocations have never been observed on their own and they always accompanied the translocation in the midpiece, this type of cell was assigned to the group with PST in the midpiece.

It has been observed that PST occurred both in morphologically normal and in abnormal forms of spermatozoa. In the fraction of vital cells with PST, there was no difference in PST prevalence between morphologically normal and morphologically abnormal spermatozoa 


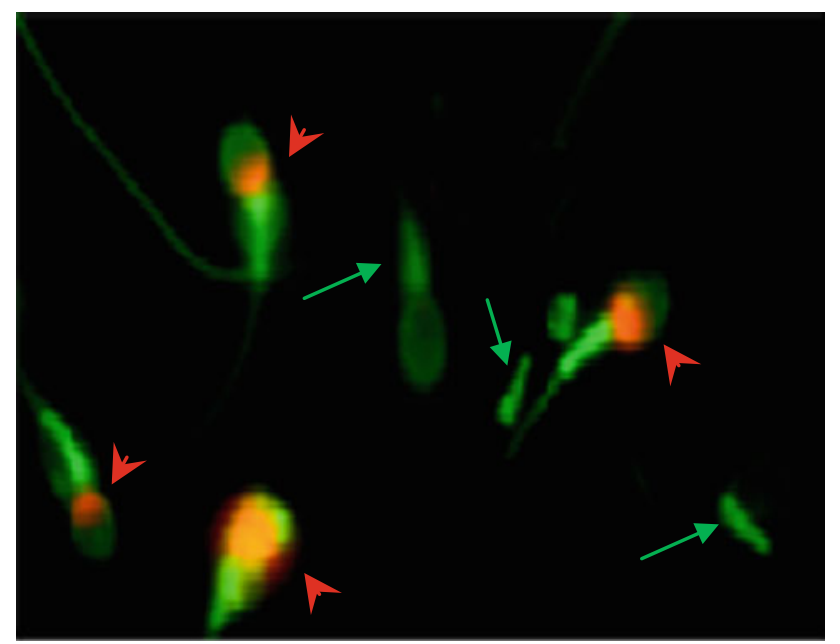

Fig. 1 Examples of spermatozoa from different fractions. Arrows indicate vital spermatozoa with $\mathrm{PS}$ translocation $\left(\mathrm{AnV}^{+} / \mathrm{PI}^{-}\right)$. Arrowheads indicate dead spermatozoa with PS translocation $\left(\mathrm{AnV}^{+} / \mathrm{PI}^{+}\right)$. Magnification $100 \times$

$(P>0.05)$. In morphologically normal spermatozoa, the region of the midpiece was the most frequent site of PST. In spermatozoa with a cytoplasmic droplet, the region of the midpiece and the region of the drop were the most evident places of AnV binding. In spermatozoa with head defects, PST took place in the head. In cells with a double head, PST was located in both heads. In spermatozoa with a double tail or midpiece defects, the region of the midpiece was the most frequent site of PST (Fig. 2).

In the fraction of vital spermatozoa susceptible to AnV binding, a significant difference between proportions of spermatozoa presenting PST located in separate sites of the cell was observed $(P<0.0001)$. In $88.5 \pm 7.2 \%$ of vital $\mathrm{AnV}^{+}$spermatozoa, PST was present in the cytomembrane of the midpiece. A multiple comparison test revealed a statistically significant difference between the proportions of vital spermatozoa with PST located in separate cell sites as follows: (1) in the midpiece vs. in the acrosomal part $(88.5 \pm 7.2 \%$ vs. $10.3 \pm 7.1 \% ; P<0.01),(2)$ in the midpiece vs. in the midpiece and the acrosomal part $(88.5 \pm 7.2 \%$ vs. $1.3 \pm 1.1 \% ; P<0.001)$ and (3) in the acrosomal part vs. in the midpiece and acrosomal part $(10.3 \pm 7.1 \%$ vs. $1.3 \pm 1.1 \% ; P<0.05)$ (Fig. $3 a)$.

In dead spermatozoa susceptible to AnV binding, simultaneous membrane PST in the midpiece and acrosomal part was most often observed. The difference between proportions of spermatozoa with PST located in separate sites of the cell was significant $(P<0.0001)$. A multiple comparison test did not reveal a significant difference between proportions of dead spermatozoa with PST in the midpiece and in the acrosomal part $(21.1 \pm 10.2 \%$ vs. $26.6 \pm 12.1 \% ; P>0.05)$. Significant differences were found between proportions of dead spermatozoa with PST in the midpiece vs. in the midpiece and acrosomal part $(21.1 \pm 10.2 \%$ vs. $52.2 \pm 15.6 \% ; P<0.001)$ and between proportions of dead spermatozoa with PST exclusively in the acrosomal part vs. in the midpiece and acrosomal part $(26.6 \pm 12.1 \%$ vs. $52.2 \pm 15.6 \% ; P<0.01)$ (Fig. 3b).

\section{Discussion}

The study revealed that semen of normozoospermic men contained spermatozoa with PST from the inner to the outer cytomembrane layer. AnV and PI staining distinguished four spermatozoa fractions: $\mathrm{AnV}^{-} / \mathrm{PI}^{-}, \mathrm{AnV}^{+} / \mathrm{PI}^{-}, \mathrm{AnV}^{-} / \mathrm{PI}^{+}$ and $\mathrm{AnV}^{+} / \mathrm{PI}^{+}$. We found that PST involved specific cytomembrane domains of spermatozoa. We noted that in vital spermatozoa PST did not occur in the acrosomal part. It is believed that protein and the lipid topography of this membrane domain are highly influenced by cytoskeletal elements formed by actin and spectrin filaments (Flesch and Gadella 2000; Dvořàkovà et al. 2005). A spectrin net binds to the cytomembrane as well as to actin filaments (An et al. 2004). It has been observed that spectrin contains aminophospholipid binding sites located in the cytomembrane cytosol layer (An et al. 2004; Cohen et al. 1986; Sikorski et al. 2000; Vance and Steenbergen 2005). It has been shown that there are PS binding sites on spectrin chains. Studies on erythrocytes revealed that a direct interaction of spectrin with cytomembrane PS has an impact on the formation of PS-rich domains (An et al. 2004). Our observations which showed very occasional PST in spectrin-rich regions, such as the acrosome and the main part of the tail, suggest that interactions between spectrin and PS are an important element responsible for retaining the asymmetrical distribution of this phospholipid.

Apart from spectrin, actin filaments are present in the acrosomal part and the main part of the tail. In human spermatozoa, actin is present predominantly in the form of a monomer, G-actin being polymerized into fibrillar form during capacitation. However, F-actin depolymerization is observed during the acrosomal reaction. It was found that actin filaments play a role in the process of aging and apoptotic death (Gourlay and Ayscough 2005), the ability of actin to bind and hydrolyze ATP being an essential feature. It is suggested that in cells in which a reduction of the ATP level is observed (e.g., as a result of mitochondrial dysfunction, hypoxia or lack of energetic substrates) the dynamics of actin filament transformation are disturbed, which leads to accumulation of F-actin aggregates (Gourlay and Ayscough 2005). Gourlay et al. (2004) suggest that the formation of F-actin aggregates may lead to cellular death by apoptosis accompanied by a decrease in mitochondrial potential, condensation of nuclear chromatin and PST. However, a precise role of actin in the activation of cellular 

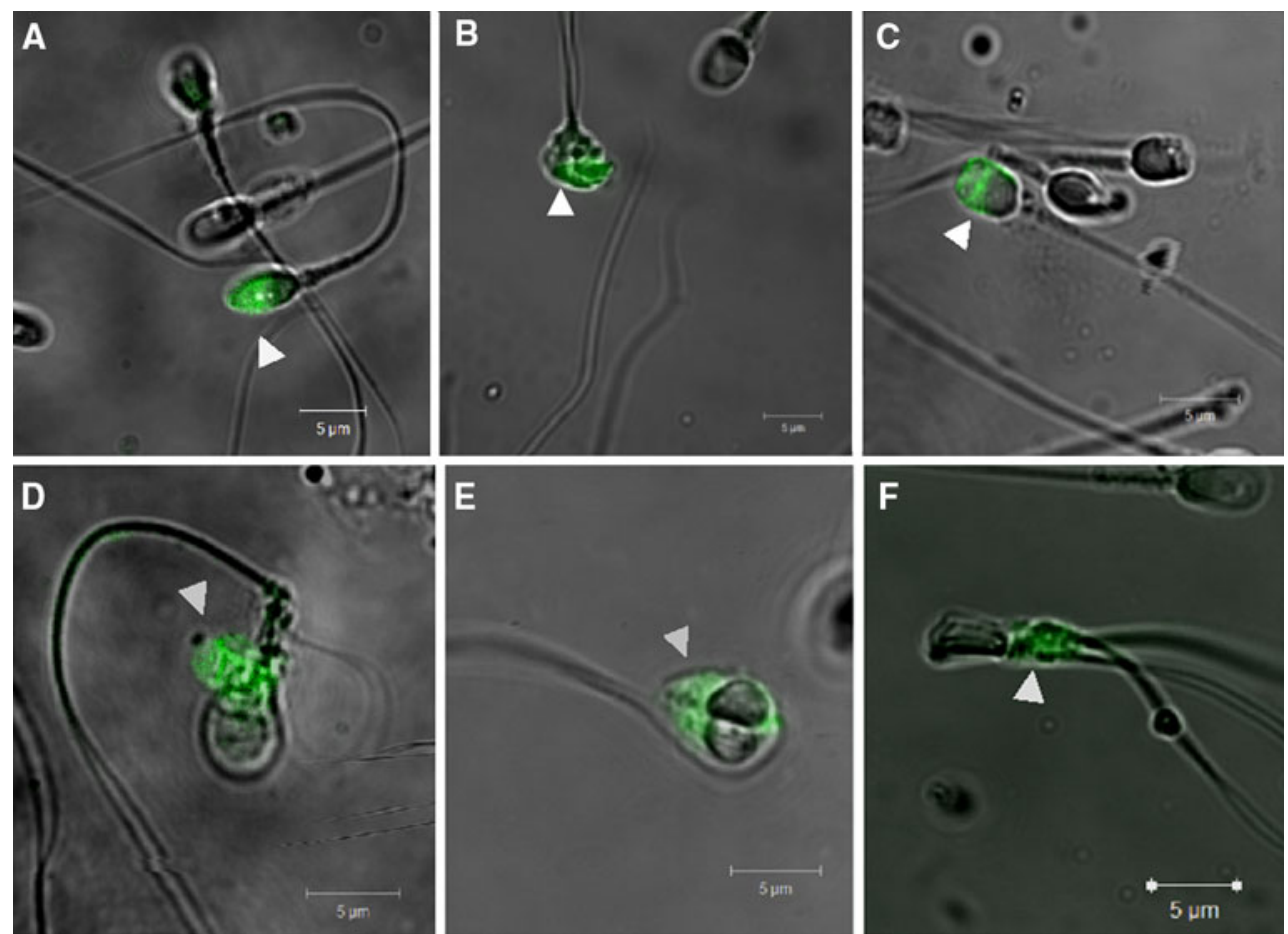

Fig. 2 Specific cell areas presenting PST in various morphological types of spermatozoa. a-c Examples of spermatozoa with head defect. d Spermatozoon with cytoplasmic droplet. e Spermatozoon with double head. f Spermatozoon with double tail. AnV-FITC staining, confocal microscope, magnification $63 x$
Fig. 3 Proportions of spermatozoa fractions according to specific area of PS membrane translocation: $\mathbf{a}$ in vital spermatozoa with PST $\left(\mathrm{AnV}^{+} /\right.$ $\left.\mathrm{PI}^{-}\right)$, $\mathbf{b}$ in dead spermatozoa with PST $\left(\mathrm{AnV}^{+} / \mathrm{PI}^{+}\right)$
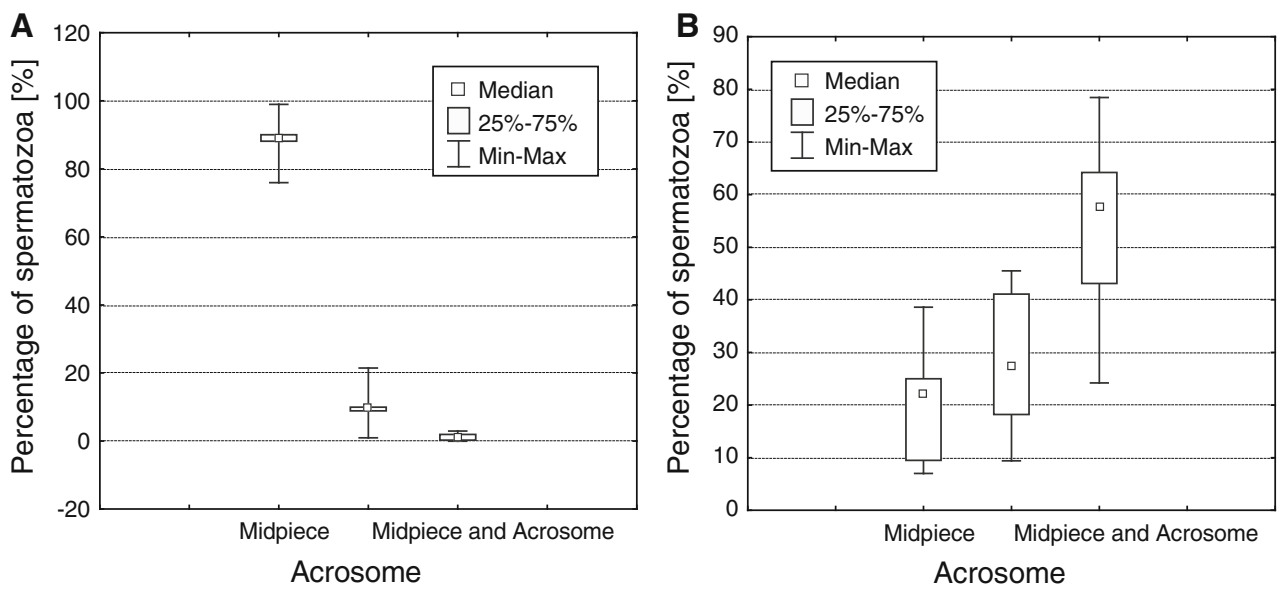

death remains unclear. The authors suggest that actin aggregates have a direct effect on mitochondrial membrane canals and, in this way, initiate apoptosis or that actin transformation disorders indirectly influence the activity of mitochondria (Gourlay and Ayscough 2005; Gourlay et al. 2004). It cannot be excluded that a decrease in the ATP concentration typical for aging spermatozoa may lead to a significant disturbance in actin transformation dynamics and a consequent disorder in the structure of spermatozoa cortex and its connections with cytomembrane lipids. An increase in acrosomal domain PST, which was observed in this study in dying spermatozoa, seems to confirm this hypothesis.
In spermatozoa, the activity of ATP-dependent aminophospholipid translocase highly contributes to the asymmetrical distribution of membrane phospholipids (Kurz et al. 2005). Studies on murine spermatozoa conducted by Wang et al. (2001) proved strong expression of this enzyme but exclusively in the acrosomal region of the cytomembrane. Our observation that in normal human spermatozoa PST rarely occurs in the acrosomal region may be connected with a high expression of flippase in this membrane domain. It is a known fact that activity of flippase depends on the ATP level, while high intracellular calcium ion concentrations inhibit activity of the enzyme. This study 
indicated that in a fraction of spermatozoa which bind PI, cells with simultaneous PST in the acrosomal part and in the midpiece are predominant. Increased intensity of PST in the acrosomal membrane domain may be a consequence of flippase activity reduction in dying or pathological cells. Müller et al. (1994) proved that in sheep spermatozoa, which bind PI, flippase activity is suppressed. Studies on erythrocytes indicate significant reduction of flippase activity in aging cells expressed by increased surface PS expression. It appears that this phenomenon is connected with a considerable decrease in ATP level and an increase in $\mathrm{Ca}^{2+}$ ion concentration. As in somatic cells, death of spermatozoa is accompanied by a calcium homeostasis disorder. It is likely that spermatozoa and erythrocytes are similar in their mechanism of inducing PS externalization by a significant increase in the calcium ion concentration. It might be expected that all dead spermatozoa (susceptible to PI staining) would demonstrate PST. It seems that the presence of a spermatozoa fraction susceptible to PI staining with no features of PST may result from a slow character of a passive phospholipid flop (Vance and Steenbergen 2005). Presumably, in some spermatozoa, first, the cytomembrane integrity is disturbed, so PI penetrates into the nucleus; only then may PST occur. Such a sequence of events would indicate a mechanism of necrotic spermatozoa death. In somatic apoptotic cells, PST takes places already at the early stages of the process; however, the cytomembrane remains impermeable to PI. Analogously, it is assumed that simultaneous $\mathrm{AnV}$ and PI staining allows for distinguishing vital $\left(\mathrm{AnV}^{-} / \mathrm{PI}^{-}\right)$apoptotic $\left(\mathrm{AnV}^{+} / \mathrm{PI}^{-}\right)$and necrotic $\left(\mathrm{AnV}^{-} / \mathrm{PI}^{+}\right.$and $\left.\mathrm{AnV}^{+} / \mathrm{PI}^{+}\right)$ spermatozoa (Ricci et al. 2002). This division is justified under the condition that membrane PST of vital spermatozoa reflects intracellular apoptosis. As we already know, not every cell with PST is an apoptotic cell. Hence, this terminology should be used carefully.

In our studies, most $\mathrm{AnV}^{+} / \mathrm{PI}^{-}$spermatozoa did not display PST in the equatorial region. Presumably, the cytomembrane of the equatorial region is stabilized by means of strong interactions between membrane components and the cytoskeleton, which in this part of a spermatozoa consists mainly of vimentin (Virtanen et al. 1984); however, these mechanisms have not been explicitly recognized.

Most vital spermatozoa ( $>88 \%$ ) in which membrane PST has been identified bind AnV exclusively in the region of the midpiece. Such a localization suggests a connection between cytomembrane PST of the midpiece and accumulation of mitochondria in this part of a cell. In some spermatozoa, PST in the midpiece was accompanied by translocation in the post-acrosomal region cytomembrane. It should be emphasized that post-acrosomal part translocation never occurred separately. It seems that the mechanism leading to PST in the midpiece cytomembrane spreads to the post-acrosomal part of the membrane, which is facilitated by the loss of membrane domain autonomy in damaged, aging and dying cells. It would seem that cytomembrane PST of the midpiece region may be influenced by oxygen free radicals or cytochrome $c$, both released from mitochondria, which may lead to translocation by inducing peroxidation of membrane phospholipids, including PS.

Our study revealed that PST occurred both in morphologically normal and in pathological spermatozoa. It has been noted that the translocation site depends on the morphology of spermatozoa. Our findings provide evidence that in most cases PST took place in the morphologically abnormal part of cells: in spermatozoa with head defects it took place in the head, in spermatozoa with tail defects it took place in the tail, in spermatozoa with a cytoplasmic droplet it occurred in the drop.

Abnormal morphology of spermatozoa frequently accompanied by changes in distribution of cytoskeletal elements may affect the cytomembrane architecture. It has been observed that in abnormal head spermatozoa there was vimentin expression in the acrosomal and post-acrosomal parts as well as the proximal part of the midpiece, while in normal spermatozoa this protein tends to occur only in the equatorial part (Marinova et al. 1996; Markova et al. 2002). Changes in cytoskeletal architecture have been identified among other findings in spermatozoa with a cytoplasmic droplet. In addition, it has been reported that cell organelles such as ribosomes and mitochondria may occur in the region of the cytoplasmic droplet (Aitken 1995). It has been reported that due to the presence of mitochondria reactive oxygen forms were produced in the cytoplasmic droplet (Aziz et al. 2007). It cannot be excluded that reactive oxygen forms produced in the drop may induce peroxidation of membrane lipids, which in turn, among other things, might lead to PST.

There are no explicit scientific data on a relationship between biochemical markers of apoptosis and morphology of spermatozoa. Said et al. (2005) did not observe any correlation between spermatozoa morphology evaluated according to WHO criteria, caspase-3 activity and PST. Similarly, Ricci et al. (2002) did not identify a correlation between the morphology and PST. However, Chen et al. (2006) reported a significant negative correlation between proportions of "apoptotic" spermatozoa (evaluated using the TUNEL method) and proportions of normal spermatozoa. Aziz et al. (2004) studied the morphology of spermatozoa with PST which had been isolated from semen using a magnetic sorting technique. The authors claim that spermatozoa with PST demonstrated a much worse morphological profile compared to spermatozoa that remained resistant to AnV binding.

In conclusion, our finding indicating a strict relation of PST to specific cell membrane domains indicates 
functional specificity of this process. Local mechanisms controling PST are to be further investigated. Since PST was observed both in morphologically normal and in abnormal cells, it seems doubtful to include this phenomenon in the physiological mechanisms of elimination of abnormal spermatozoa.

Open Access This article is distributed under the terms of the Creative Commons Attribution Noncommercial License which permits any noncommercial use, distribution, and reproduction in any medium, provided the original author(s) and source are credited.

\section{References}

Aitken J (1995) Cell biology of human spermatozoa. In: Grudzinskas JG, Yovich JL (eds) Gametes: the spermatozoon. Cambridge University Press, Cambridge, pp 206-238

An X, Guo X, Wu Y et al (2004) Phosphatidylserine binding sites in red cell spectrin. Blood Cells Mol Dis 32:430-432

Aziz N, Saleh RA, Sharma RK et al (2004) Novel association between sperm reactive oxygen species production, sperm morphological defects, and the sperm deformity index. Fertil Steril 81:349-354

Aziz N, Said T, Paasch U et al (2007) The relationship between human sperm apoptosis, morphology and the sperm deformity index. Hum Reprod 22:1413-1419

Chen Z, Hauser R, Trbovich AM et al (2006) The relationship between human semen characteristics and sperm apoptosis: a pilot study. J Androl 27:112-120

Cohen AM, Liu SC, Derick LH et al (1986) Ultrastructural studies of the interaction of spectrin with phosphatidylserine liposomes. Blood 68:920-926

Curry MR, Watson PF (1995) Sperm structure and function. In: Grudzinskas JG, Yovich JL (eds) Gametes: the spermatozoon. Cambridge University Press, Cambridge, pp 45-70

Dvořàkovà K, Moore DM, Šebkovà N et al (2005) Cytoskeleton localization in the sperm head prior to fertilization. Reproduction 130:61-69

Flesch FM, Gadella BM (2000) Dynamics of the mammalian sperm plasma membrane in the process of fertilization. Biochem Biophys Acta 1469:197-235

Gourlay CW, Ayscough KR (2005) The actin cytoskeleton: a key regulator of apoptosis and ageing? Nat Rev 6:583-589
Gourlay CW, Carpp LN, Timpson P et al (2004) A role for the actin cytoskeleton in cell death and aging in yeast. J Cell Biol 164:803-809

Kurz A, Viertel D, Herrmann A et al (2005) Localization of phosphatidylserine in boar spermatozoa membranes during capacitation and acrosome reaction. Reproduction 130:615-626

Ladha S, James PS, Clark DC et al (1997) Lateral mobility of plasma membrane lipids in bull spermatozoa: heterogeneity between surface domains and rigidification following cell death. J Cell Sci 110:1041-1050

Marinova TT, Markova M, Stanislavov RK (1996) Distribution of vimentin in abnormal human spermatozoa. Andrology 28:287289

Markova MD, Marinova TT, Vatev IT (2002) Asymmetric vimentin distribution in human spermatozoa. Folia Biol 48:160-162

Müller K, Pomorski T, Müller P et al (1994) Protein-dependent translocation of aminophospholipids and asymmetric transbilayer distribution of phospholipids in the plasma membrane of ram spermatozoa. Biochemistry 33:9968-9974

Ricci G, Perticari S, Fragonas E et al (2002) Apoptosis in human sperm: its correlation with semen quality and the presence of leukocytes. Hum Reprod 17:2665-2672

Said TM, Grunewald S, Paasch U et al (2005) Advantage of combining magnetic cell separation with sperm preparation techniques. Reprod Biomed Online 10:740-746

Shadan S, James PS, Howes EA et al (2004) Cholesterol efflux alters lipid raft stability and distribution during capacitation of boar spermatozoa. Biol Reprod 71:253-265

Sikorski AF, Hanus-Lorenz B, Jezierski A et al (2000) Interaction of membrane skeletal proteins with membrane lipid domain. Acta Biochim Pol 47:565-578

Vance JC, Steenbergen R (2005) Metabolism and functions of phosphatidylserine. Prog Lipid Res 44:207-234

Virtanen I, Badley A, Paasivuo R et al (1984) Distinct cytoskeletal domains revealed in spermatozoa. J Cell Biol 99:1083-1091

Wang Y, Storeng R, Dale PO et al (2001) Effects of follicular fluid and steroid hormones on chemotaxis and motility of human spermatozoa in vitro. Gynecol Endocrinol 15:286-292

Wolfe CA, James PS, Mackie AR et al (1998) Regionalized lipid diffusion in the plasma membrane of mammalian spermatozoa. Biol Reprod 59:1506-1514

World Health Organization (1999) WHO laboratory manual for the examination of human semen and sperm-cervical mucus interaction. Cambridge University Press, Cambridge 